\title{
Improving patient recall of information: Harnessing the power of structure
}

\author{
Wolf Langewitz $^{\mathrm{a}, *}$, Selina Ackermann ${ }^{\mathrm{b}}$, Anette Heierle ${ }^{\mathrm{b}}$, Ralph Hertwig ${ }^{\mathrm{c}}$, Leyla Ghanim ${ }^{\mathrm{d}}$, \\ Roland Bingisser ${ }^{\mathrm{b}}$
}

${ }^{a}$ Department of Psychosomatic Medicine, University Hospital Basel, Basel, Switzerland

${ }^{\mathrm{b}}$ Department of Emergency Medicine, University Hospital Basel, Basel, Switzerland

${ }^{\mathrm{c}}$ Center for Adaptive Rationality, Max Planck Institute for Human Development, Berlin, Germany

d Medical University Graz, Graz, Austria

\section{A R T I C L E I N F O}

\section{Article history:}

Received 2 July 2014

Received in revised form 20 January 2015

Accepted 7 February 2015

\section{Keywords:}

Patient information

Information recall

Improving recall

Structuring information

Book metaphor

\begin{abstract}
A B S T R A C T
Objective: Assess the amount of medical information laypeople recall, investigate the impact of structured presentation on recall.

Methods: 105 first-year psychology students (mean age $21.5 \pm 3.8$ years; $85 \%$ female) were randomised to two information-presentation conditions: structured (S group) and nonstructured (NS group). Students watched a video of a physician discharging a patient from the emergency department. In the S Group, content (28 items of information) was divided into explicit "chapters" with "chapter headings" preceding new information. Afterwards, participants wrote down all information they recalled on an empty sheet of paper. Results: The $S$ group $(N=57)$ recalled significantly more items than NS group $(N=41)(8.12 \pm 4.31$ vs. $5.71 \pm 3.73 ; p=0.005)$, rated information as easier to understand $(8.0 \pm 1.9$ vs. $6.1 \pm 2.2 ; p<0.001)$ and better structured $(8.5 \pm 1.5$ vs. $5.5 \pm 2.7 ; p<0.001)$; they rather recommended the physician to friends $(7.1 \pm 2.7$ vs. $5.8 \pm 2.6 ; p<0.01)$.

Conclusion: University students recalled around 7/28 items of information presented. Explicit structure improved recall.

Practice implications: Practitioners must reduce the amount of information conveyed and structure information to improve recall.
\end{abstract}

() 2015 Elsevier Ireland Ltd. All rights reserved.

\section{Introduction}

Communication in health care often means the exchange of medical information. This is true for ward rounds in internal medicine [1], outpatients in internal medicine [2], and oncological consultations (e.g. $[3,4])$. Findings indicate that many patients and their relatives want to be fully informed about their condition [5-8]. For instance, questionnaire data indicate that $87 \%$ of patients "want to be told all information" and only $9 \%$ "want the doctor to choose how much information to give" [9] (see also [5,6]). Similarly, qualitative data show that both patients and parents

\footnotetext{
* Corresponding author at: University Hospital Basel, Department of Psychosomatic Medicine, Hebelstr. 2, CH-4031 Basel, Switzerland. Tel.: +41 6126553 18; fax: +416126532 28 .

E-mail addresses: wolf.langewitz@usb.ch (W. Langewitz),

selina.ackermann@usb.ch (S. Ackermann), anette.heierle@usb.ch (A. Heierle), sekhertwig@mpib-berlin.mpg.de (R. Hertwig), leyla.ghanim@medunigraz.at

(L. Ghanim), roland.bingisser@usb.ch (R. Bingisser).
}

expect physicians to inform them about diagnosis, therapy, and prognosis [10-12].

Patient-physician communication goes beyond the filling of knowledge gaps, however. It is also the basis for patients' inferences about the health practitioner. For instance, recent qualitative studies on communication in oncology have demonstrated that patients' trust is based primarily on the impression of clinical competence that emerges from their communication with oncological surgeons and haematologists [13]. Furthermore, Parker et al. [14] and Hagerty et al. [15] have reported that patients' hope depends largely on the impression that their physician is competent and "knows all there is to know about the disease". Physicians may not be aware of the importance that patients attribute to receiving information, however: In their studies of patient centeredness and consultation skills in primary care, Ogden et al. [7] and Robinson et al. [8] found that patients ranked items relating to patient information and the structure of consultations significantly higher than physicians did. 
However, other findings suggest that the exchange of information may be an ephemeral phenomenon. Specifically, several studies have found that patient comprehension and recall of information is limited [16,17]. Three examples from surgery illustrate these limitations: On average, only 2 out of 5 complications were recalled in the context of elective plastic surgery [18]; 5 out of 32 pieces of information were correctly remembered $2 \mathrm{~h}$ after the preoperative discussion prior to brain surgery and 4 out of 25 pieces of information prior to spinal surgery [19] (for a recent review, see [20]). Questionnaire data from patients with advanced metastatic cancer revealed how little patients understood of their clinical situation. Although they had been informed by their doctors about the advanced stage of their disease and the clinical consequences, they largely overestimated the chance of recovery and failed to understand the palliative rather than curative goal of their treatment [21].

These insights raise the following questions: How can patient recall and understanding of medical information be improved? One of the first authors to address these questions was Ley (e.g. [22]). Ley recommended using explicit categorisation, with the clinician presenting "information in categories, which he has announced in advance". Several review articles have since investigated whether patient understanding and recall of information can be improved by the use of additional communicative aids. Although results have been mixed and the evidence is not always convincing, the general picture to emerge is that patients recall slightly more information when they are given written or otherwise designed information material. For example, Ciciriello et al. [23] found weak evidence that the addition of multimedia material to standard instructions improved patient knowledge about medication (see also [24-26]). To our knowledge, however, none of the interventions evaluated in these review articles have focused on the explicit structuring of verbal information.

Although the provision of generic written information improves patient knowledge to some extent, it is associated with two major problems: First, information leaflets on diagnostic interventions usually cover the standard procedure in common diagnoses. However, the typical patient presents with a more complex combination of symptoms, diagnoses, and treatment options - a complexity that cannot be accommodated in standardised materials. Second, even when provided with the most sophisticated information material, patients show much lower recall capacity than physicians evidently assume: Physicians asked which information was essential for patients discharged from the emergency department after presenting with acute chest pain on average chose 36 out of 81 pre-defined items [27] - far beyond the typical recall capacity reported in the literature (e.g. [20]). Both problems are related. Tailoring information to more complex real-world cases is likely to involve the provision of even more information.

In principle, there are two ways out of this dilemma: less information or better communication. By better communication, we mean communication in ways that increase the likelihood that patients will later be able to retrieve the information. Here, we investigate whether structuring medical information improves recall. Specifically, information appears easier to retain when it is structured in a way that helps the recipient to organise it $[28,29]$. In written material, structure is reflected in the way content is ordered sequentially. For instance, in newspapers, headlines precede the main text and are easy to identify; they announce the topic elaborated on in the text. Books use even more sophisticated structural elements to guide readers through content: title, table of contents, chapter headings, text, reference list, etc. In our communication skills training for medical students, we have used the term "book metaphor" to help participants understand, appreciate and remember the value and function of "structuring information" [30,31].
In this pilot study, we investigated whether first-year psychology students serving as surrogate patients recalled more information when discharge information was presented in structured form, in accordance with the book metaphor, than they did when exactly the same information was presented in nonstructured form.

\section{Methods}

\subsection{Participants}

First-year psychology students were invited to participate in a trial measuring recall of medical information. Of the 167 students approached, 105 agreed to participate and provided informed consent. Sixteen of these students were male; mean age was $21.5 \pm 3.8$ years. Ninety-eight students returned completed recall protocols. The study was approved by the local ethics committee (protocol number: 362/11). Participants received no compensation for their participation.

\subsection{Study design}

On their arrival, students were randomly allocated to two lecture halls. They were informed that they were participating in an experiment about physicians' communication style, and that they would be shown a video of a physician discharging a patient from the emergency department. The patient was a white man of around 75 years of age, played by an actor. The information conveyed was defined after a careful Delphi process, in which three expert physicians agreed on 28 items of information that they considered essential for a patient with unstable angina pectoris after exclusion of acute myocardial infarction [32]. The experts were informed that this information would be given to a patient during discharge from the emergency department, that the time allotted for this consultation was a maximum of $15 \mathrm{~min}$, and that the patient would see his GP within the next two or three days. Study authors reviewed the two versions of the video to make sure that both contained the same factual information.

The two student groups watched the same male physician deliver exactly the same 28 items of information in a friendly manner and without the use of medical jargon in either structured or nonstructured form. Specifically, in the nonstructured condition (NS group), the order of presentation was based on traditional clinical wisdom: pieces of information that belonged together because they pertained to, for example, the likely diagnosis of coronary artery disease were presented in one block of information (likewise, there were blocks of information on pathophysiology, further work-up, therapy, and red flags). However, there was no explicit structure. In the structured condition ( $\mathrm{S}$ group) the information presented was structured following the structural elements of a book, in which the content is presented in a specific order, typically advancing from summary, high-level information (e.g., title, table of contents, chapter headings) to detailed, lowlevel information (e.g., text, annexes). Following this book structure [33], the physician initiated the interaction as follows:

Mr. Lehmann, I will now give you some discharge information (TITLE)

Before you go home, there are five points that I would like to inform you about (TABLE OF CONTENTS)

First: What is your diagnosis?

Second: What will happen next?

Third: What can you do yourself? 
Fourth: What do you have to pay attention to in order to be on the safe side?

Finally, the fifth and last point: What will the treatment look like? Let me start with the first point: What is your diagnosis (1st CHAPTER HEADING)

The good news is that you don't have a myocardial infarction .... (TEXT)

\subsection{Dependent variables}

Immediately after watching the video (and without prior warning), students were given $5 \mathrm{~min}$ to write down all the information they remembered from the exchange. They were asked not to consult their neighbours. No further instructions were given. Students were then asked to complete a questionnaire assessing their prior medical knowledge. This multiple-choice questionnaire covered the following topics: definition of myocardial infarction; definition of angina pectoris; risk factors for cardiovascular disease; typical pain sensations in myocardial infarction; cardiac angiography; physiological processes typically associated with cardiac pain. They then rated their current sense of well-being (numerical rating scale from 0 [very bad] to 10 [very good]), their ability to concentrate on the day of the study (numerical rating scale from 0 [very low] to 10 [very high]), and their perception of the physician on three items ranging from 0 to 10 , with 0 representing a low or negative response and 10 representing a high or positive response.

Students' recall performance (i.e., the number of items of information recalled) was assessed by two independent raters, one of whom rated all of the protocols and the other, $10 \%$ of them. Analyses of the agreement between the two raters resulted in a Cohen's kappa of 0.74 , indicating substantial interrater reliability according to Landis and Koch [34]. When it became clear that some students in the structured condition had also noted down chapter headings, we also assessed the extent to which students recalled these structuring elements. Both raters independently screened all protocols to identify the five chapter headings listed in the example above. They agreed fully on the number of protocols in which at least one chapter heading was recalled and differed in the number of chapter headings recalled in only 2 of those 33 cases. Agreement was achieved by discussing these differences.

\subsection{Statistical analysis}

All data were analysed with SPSS. Items recall is presented in terms of the percentage of students who noted down each item in the recall protocol. We report means and standard deviations for students' subjective well-being, concentration ability, and perceptions of the physician in the two conditions; comparisons between groups were conducted using $t$-tests for independent samples. The influence of prior medical knowledge, subjective well-being, and concentration ability on recall was assessed by an analysis of covariance (ANCOVA), with number of recalled items as the dependent variable and condition as the independent variable.

\section{Results}

One student in the nonstructured and 6 students in the structured condition returned empty recall protocols. Students in both groups reported a similar sense of well-being on the day of the study $(6.5 \pm 1.8$ in both groups; n.s.). Students in the structured group felt better able to concentrate $(6.5 \pm 1.8$ vs. $5.6 \pm 2.0 ; p=0.03)$. Students in the structured group answered $3.20 \pm 1.3$ items correctly in the medical knowledge assessment, slightly but not significantly more than their counterparts in the nonstructured group $(2.94 \pm 1.08$ items, n.s.).

Table 1 presents both groups' perception of the physician, showing more positive responses in the structured group.

The mean number of items recalled was $8.12 \pm 4.31(N=57)$ in the structured condition and $5.71 \pm 3.73(N=41)$ in the nonstructured condition $(p=0.005)$. The mean for the whole sample was $7.11 \pm 4.23$ items. Table 2 lists the percentage of participants who correctly recalled each item. Of the 57 participants in the structured condition, 33 spontaneously listed $3.45 \pm 1.23$ chapter headings in addition to factual items. Students who did not list chapter headings recalled $8.33 \pm 4.4$ items; those who did recalled $7.97 \pm 4.27$ items (n.s.). None of the covariates (prior medical knowledge, age, gender, sense of well-being, ability to concentrate, perception of the physician) influenced the number of items recalled.

\section{Discussion and conclusion}

\subsection{Discussion}

Our findings show that the number of items of information that experts considered essential for patients being discharged from the emergency department by far exceeded participants' recall capacity [18]. This finding could have major consequences for clinical practice and teaching. Assuming that medical information has one primary goal, namely to enable the patient to make informed choices, the elements of information provided need to be limited to a number that patients can retain in memory and use during the decision-making process. In other words, the amount of oral information transmitted needs to be drastically reduced.

So how can health professionals identify the elements that are truly essential for a patient to know? In the context of our study, information on red flags signalling that the patient should return to the emergency department is essential because it might save a patient's life. Another crucial piece of information is arguably how to prevent another vascular event by adhering to prescribed drugs and dosages. Yet our results showed that information on red flags and medication was not well recalled [35].

Table 1

Secondary outcome: perception of the doctor in both groups.

\begin{tabular}{|c|c|c|c|c|}
\hline Group & & $\begin{array}{l}\text { How well understandable } \\
\text { was the information? }(0-10)\end{array}$ & $\begin{array}{l}\text { How well } \\
\text { structured was } \\
\text { the sequence of } \\
\text { information items? }(0-10)\end{array}$ & $\begin{array}{l}\text { Would you } \\
\text { recommend this } \\
\text { physician to your } \\
\text { friends? }(0-10)\end{array}$ \\
\hline \multirow[t]{3}{*}{ Structured information } & $N$ & 57 & 57 & 57 \\
\hline & Mean & 8.0 & 8.5 & 7.1 \\
\hline & SD & 1.9 & 1.5 & 2.7 \\
\hline \multirow[t]{4}{*}{ Unstructured information } & $N$ & 41 & 41 & 41 \\
\hline & Mean & 6.1 & 5.5 & 5.8 \\
\hline & SD & 2.2 & 2.7 & 2.6 \\
\hline & & $p<0.001$ & $p<0.001$ & $p<0.01$ \\
\hline
\end{tabular}


Table 2

Percent of items recalled in the unstructured and the structured information group.

\begin{tabular}{|c|c|c|}
\hline Item & $\begin{array}{l}\text { \% recalled } \\
\text { structured } \\
\text { information }\end{array}$ & $\begin{array}{l}\% \text { recalled } \\
\text { unstructured } \\
\text { information }\end{array}$ \\
\hline Patient can go home & 11 & 20 \\
\hline Patient was reassured ("you were right to come here") & 4 & $15^{*}$ \\
\hline $\begin{array}{l}\text { Presumptive diagnosis of Angina Pectoris (narrowing of } \\
\text { cardiac blood vessels) }\end{array}$ & $51^{* * *}$ & 10 \\
\hline Right now it is no myocardial infarction & 47 & $76^{* *}$ \\
\hline Narrowing of cardiac vessels means problems with oxygen supply & 65 & 59 \\
\hline Narrowing of cardiac vessels means avoid strenuous exercise & 18 & 07 \\
\hline Further investigation clarifies the extent of narrowing & 35 & 29 \\
\hline Myocardial scintigraphy will be performed & $14^{*}$ & 0 \\
\hline Myocardial scintigraphy will be performed next week & 26 & 27 \\
\hline Myocardial scintigraphy will be done here in hospital & 11 & 5 \\
\hline No coffee, no tea, no chocolate prior to myocardial scintigraphy & $35^{* *}$ & 12 \\
\hline Information on time and location of the test will be sent by post & 11 & 7 \\
\hline $\begin{array}{l}\text { Patient is advised to contact his/her family physician should he have } \\
\text { further questions }\end{array}$ & 5 & 2 \\
\hline Patient was told to try abstain from smoking & 60 & 51 \\
\hline Patient was told to avoid physical stress & $51^{* * *}$ & 15 \\
\hline Patient was told to come to the ED in case of chest-pain radiating into arms/jaws & 49 & 46 \\
\hline Patient was told to present immediately to the ED if symptoms lasted longer than $10 \mathrm{~min}$ & 19 & 12 \\
\hline Patient was told to present immediately to the ED if he was dyspnoeic & 4 & 7 \\
\hline $\begin{array}{l}\text { Patient was told to present immediately to the ED if he experienced chest } \\
\text { pain not responding to nitroglycerine }\end{array}$ & 11 & 17 \\
\hline Patient was informed that the ED is open $24 / 7$, also at night & 35 & 41 \\
\hline Patient was told that treatment needs to start immediately & 5 & $22^{*}$ \\
\hline Patient was told why treatment needs to start immediately & 5 & 15 \\
\hline Name of the new medication was given: Aspirin & $53^{* * *}$ & 10 \\
\hline Name of the new medication was given: Beloc (Betablocker) & $16^{*}$ & 2 \\
\hline Name of the new medication was given: Nitro-Spray & $70^{* * *}$ & 20 \\
\hline ASS dose and mode of intake & $26^{*}$ & 10 \\
\hline B-Blocker dose and mode of intake & 19 & 7 \\
\hline Nitroglycerine dose and mode of intake & $58^{*}$ & 37 \\
\hline
\end{tabular}

Significant differences are shown with ${ }^{*}(p<0.05),{ }^{* *}(p<0.01),{ }^{* * *}(p<0.001)$.

A second major result is that a simple communication technique improves recall of medical information. To our knowledge, this is the first cognitive intervention in a clinical setting to implement the suggestions of authors such as Doak and Doak [29] and Ley (e.g. [22]), who argued that structured information is easier to recall than nonstructured information. However, these authors did not provide strong evidence to support this hypothesis. Studies with chess players have convincingly demonstrated that people retrieve information much better when it is organised along familiar structures: master chess players recalled the position of pieces in a chess game with an incredible precision of $93 \%$ after a presentation time of only $5 \mathrm{~s}$ [36], and fared much worse when they were asked to recall the position of randomly placed pieces [37].

One might argue that recall in the present study would have been better if participants had been given longer to complete their recall protocols. We doubt this to be the case, because almost all participants finished within $5 \mathrm{~min}$ in a pre-pilot test. Even if the time allotted were too short, this would primarily have disadvantaged students in the structured condition, where there was more information to be recalled, namely, chapter headings in addition to factual information.

Indeed, the observation that many students in the structured group spontaneously wrote down chapter headings in addition to factual items was an interesting and unexpected finding. These students apparently dealt with chapter headings in a similar way as they did single items of factual information. One might argue that the imposition of structure renders necessary the provision of more elements of information, with detrimental effects on recallspecifically, that increased memory load decreases the likelihood of recall of target items in the structured condition. However, we showed, first, that structure improved recall of target items and, second, that there was no difference in the number of factual items recalled by students who wrote down chapter headings and those who did not. Therefore, the price of structure - even more information - is a price worth paying.

It seems likely that the better recall of students in the structured condition can be attributed to "chunking": the ability to form highlevel clusters of information from low-level individual elements [38-40]. The concept of a "chunk" referring to a pattern of other symbols has been studied as a model of memory organization. It has, for example, been used to explain why more elaborate prior knowledge can lead to an increased ability to extract information from the environment [38].

The recall of chapter headings or a table of contents in addition to single items may be of specific importance because it can help patients to define gaps in their knowledge. For example, a patient discharged from the emergency department may later tell his partner: "Then she told me something about the treatment plan, but I don't actually remember what she said." This patient would be better prepared to prompt the physician to repeat the information subsumed under this heading at their next encounter.

\subsection{Limitations}

Roughly two-thirds of the first-year psychology students approached responded to our invitation to participate in the present study. We do not know whether selection bias had any influence on recall. If one assumes that respondents are better motivated than nonrespondents, it would mean that our results tend to overestimate recall in a less motivated population. Along similar lines, one might argue that a sample of young psychology students is not representative of the average patient newly diagnosed with coronary heart disease. The question is whether this mismatch induced a bias in favour of the intervention, or whether the results should be interpreted with special caution. 
First-year psychology students are probably better trained and capable of storing new information than the typical 60-year-old with less formal education. Therefore, our results might in fact overestimate the number of items that typical patients are able to recall. On the other hand, it is possible that students are less motivated than real patients to recall information because they are not directly affected by it.

It is difficult to predict whether the book structure will be of more or less help to real patients than to well-educated young students, but it seems reasonable to assume that the less prior knowledge an individual has, the more helpful the combination of single items with structure will be. Thus, a patient who is able to link new information to existing knowledge or to organise new information along logical or associative strings will have better recall. The present results pertain primarily to patients with little or no prior knowledge of their condition. This generally holds for patients in an emergency situation, but may not apply to patients with chronic conditions, who consult for the same problem repeatedly and themselves acquire a continuously growing medical knowledge. Future research needs to examine whether, for example, more knowledgeable students recall more items and reap less benefit from the structuring of information.

\subsection{Conclusions}

A necessary condition for shared decision making in health care is informed patients who are knowledgeable about the consequences of a given diagnosis or a certain treatment. In order to make informed decisions, patients must be able to store information in memory and recall it when necessary. On average, study participants recalled 7 of a total of 28 items of information that were deemed essential by expert physicians. Structuring information according to the book metaphor improved recall from 5.7 items in the nonstructured condition to 8.1 items in the structured condition.

\subsection{Practice implications}

Two findings merit special attention: First, if patients recall about 7 new items (as our findings suggest), it is likely that all patients are overwhelmed by information in typical patientphysician communication. Clinicians must therefore decide which information is absolutely crucial and which information can be tailored to the patient's individual needs. This calls for action on the clinicians' part: In addition to the development of extensive information material, a consensus must be reached on which information on a given disease, diagnosis or treatment is truly essential. Second, more research is needed on the merits and limits of the book metaphor, and on the efficacy of training programs in the use of this technique.

\section{Acknowledgements}

Many thanks to Claudia Steiner and Susannah Goss for editing the manuscript and to the students who volunteered as participants. The research was supported by the Scientific Fundof the Emergency Department, University Hospital Basel.

\section{References}

[1] Weber H, Stockli M, Nubling M, Langewitz WA. Communication during ward rounds in internal medicine. An analysis of patient-nurse-physician interactions using RIAS. Patient Educ Couns 2007;67:343-8.

[2] Langewitz WA, Loeb Y, Nubling M, Hunziker S. From patient talk to physician notes-comparing the content of medical interviews with medical records in a sample of outpatients in internal medicine. Patient Educ Couns 2009;76: 336-40.
[3] Butow PN, Dowsett S, Hagerty R, Tattersall MH. Communicating prognosis to patients with metastatic disease: what do they really want to know? Support Care Cancer 2002;10:161-8 (Official journal of the Multinational Association of Supportive Care in Cancer).

[4] Butow PN, Brown RF, Cogar S, Tattersall MH, Dunn SM. Oncologists' reactions to cancer patients' verbal cues. Psychooncology 2002;11:47-58.

[5] Kaplowitz SA, Campo S, Chiu WT. Cancer patients' desires for communication of prognosis information. Health Commun 2002;14:221-41.

[6] Mack JW, Wolfe J, Grier HE, Cleary PD, Weeks JC. Communication about prognosis between parents and physicians of children with cancer: parent preferences and the impact of prognostic information. J Clin Oncol 2006;24:5265-70.

[7] Ogden J, Ambrose L, Khadra A, Manthri S, Symons L, Vass A, et al. A questionnaire study of GPs' and patients' beliefs about the different components of patient centredness. Patient Educ Couns 2002;47:223-7.

[8] Robinson J, Walley T, Pearson M, Taylor D, Barton S. Measuring consultation skills in primary care in England: evaluation and development of content of the MAAS scale. Br J Gen Pract 2002;52:889-93.

[9] Cox A, Jenkins V, Catt S, Langridge C, Fallowfield L. Information needs and experiences: an audit of UK cancer patients. Eur J Oncol Nurs 2006;10:263-72.

[10] Young B, Ward J, Forsey M, Gravenhorst K, Salmon P. Examining the validity of the unitary theory of clinical relationships: comparison of observed and experienced parent-doctor interaction. Patient Educ Couns 2011:85:60-7.

[11] Salmon P, Mendick N, Young B. Integrative qualitative communication analysis of consultation and patient and practitioner perspectives: towards a theory of authentic caring in clinical relationships. Patient Educ Couns 2011;82:448-54.

[12] Forsey M, Salmon P, Eden T, Young B. Comparing doctors' and nurses' accounts of how they provide emotional care for parents of children with acute lymphoblastic leukaemia. Psychooncology 2013:22:260-7.

[13] Kowalski C, Nitzsche A, Scheibler F, Steffen P, Albert US, Pfaff H. Breast cancer patients' trust in physicians: the impact of patients' perception of physicians' communication behaviors and hospital organizational climate. Patient Educ Couns 2009;77:344-8.

[14] Parker PA, Baile WF, de Moor C, Lenzi R, Kudelka AP, Cohen L. Breaking bad news about cancer: patients' preferences for communication. J Clin Oncol 2001:19:2049-56.

[15] Hagerty RG, Butow PN, Ellis PM, Lobb EA, Pendlebury SC, Leighl N, et al. Communicating with realism and hope: incurable cancer patients' views on the disclosure of prognosis. J Clin Oncol 2005;23:1278-88.

[16] Mulsow JJ, Feeley TM, Tierney S. Beyond consent-improving understanding in surgical patients. Am J Surg 2012;203:112-20.

[17] Kessels RP. Patients' memory for medical information. J R Soc Med 2003:96:219-22.

[18] Makdessian AS, Ellis DA, Irish JC. Informed consent in facial plastic surgery: effectiveness of a simple educational intervention. Arch Facial Plast Surg 2004;6:26-30.

[19] Krupp W, Spanehl O, Laubach W, Seifert V. Informed consent in neurosurgery: patients' recall of preoperative discussion. Acta Neurochir (Wien) 2000;142:233-8 (Discussion 8-9).

[20] Kinnersley P, Phillips K, Savage K, Kelly MJ, Farrell E, Morgan B, et al. Interventions to promote informed consent for patients undergoing surgical and other invasive healthcare procedures. Cochrane Database Syst Rev (Online) 2013; 7:CD009445.

[21] Weeks JC, Catalano PJ, Cronin A, Finkelman MD, Mack JW, Keating NL, et al. Patients' expectations about effects of chemotherapy for advanced cancer. N Engl J Med 2012;367:1616-25.

[22] Ley P. Memory for medical information. Br J Clin Psychol 1979:18:245-55.

[23] Ciciriello S, Johnston RV, Osborne RH, Wicks I, deKroo T, Clerehan R, et al. Multimedia educational interventions for consumers about prescribed and over-the-counter medications. Cochrane Database Syst Rev (Online) 2013:4:CD008416.

[24] van der Meulen N, Jansen J, van Dulmen S, Bensing J, van Weert J. Interventions to improve recall of medical information in cancer patients: a systematic review of the literature. Psychooncology 2008;17:857-68.

[25] Schenker Y, Fernandez A, Sudore R, Schillinger D. Interventions to improve patient comprehension in informed consent for medical and surgical procedures: a systematic review. Med Decis Making 2011;31:151-73.

[26] Edwards AG, Naik G, Ahmed H, Elwyn GJ, Pickles T, Hood K, et al. Personalised risk communication for informed decision making about taking screening tests. Cochrane Database Syst Rev (Online) 2013;2 CD001865.

[27] Ackermann S, Bingisser M-B, Heierle A, Langewitz W, Hertwig R, Bingisser R. Discharge communication in the emergency department: physicians underestimate the time needed. Swiss Med Wkly 2012;142:1-20.

[28] van Merrienboer JJ, Sweller J. Cognitive load theory in health professional education: design principles and strategies. Med Educ 2010;44:85-93.

[29] Doak CC, Doak LG, Friedell GH, Meade CD. Improving comprehension for cancer patients with low literacy skills: strategies for clinicians. CA Cancer J Clin 1998;48:151-62.

[30] Kiessling C, Langewitz W. The longitudinal curriculum social and communicative competencies within Bologna-reformed undergraduate medical education in Basel. GMS Z Med. Ausbildung 2013;30 (Doc31).

[31] Schweizerische Akademie der Medizinischen Wissenschaften. Kommunikation im medizinischen Alltag. Ein Leitfaden für die Praxis. Basel SAMW; 2013 
[32] Ackermann S, Bingisser MB, Heierle A, Langewitz W, Hertwig R, Bingisser R. Discharge communication in the emergency department: physicians underestimate the time needed. Swiss Med Wkly 2012;142:w13588.

[33] Langewitz W. Zur Erlernbarkeit der arzt-patienten-kommunikation in der medizinischen ausbildung. Bundesgesundheitsblatt 2012;55:1176-82.

[34] Landis JR, Koch GG. The measurement of observer agreement for categorical data. Biometrics 1977;33:159-74.

[35] Donner-Banzhoff N, Hertwig R. Inductive foraging: improving the diagnostic yield of primary care consultations. Eur J Gen Pract 2014;20:69-73.

[36] De Groot AD. Thought and choice in chess. The Hague: Mouton Publishers; 1965
[37] Gobet F, Simon HA. Templates in chess memory: a mechanism for recalling several boards. Cognit Psychol 1996;31:1-40.

[38] Gobet F, Lane PC, Croker S, Cheng PC, Jones G, Oliver I, et al. Chunking mechanisms in human learning. Trends Cogn Sci 2001;5:236-43.

[39] Chen Z, Cowan N. Chunk limits and length limits in immediate recall: a reconciliation. J Exp Psychol Learn Mem Cogn 2005;31:1235-49.

[40] Li G, Ning N, Ramanathan K, He W, Pan L, Shi L. Behind the magical numbers: hierarchical chunking and the human working memory capacity. Int J Neural Syst 2013;23:1350019. 